\title{
Cooperation enhances structural stability in mutualistic systems
}

\author{
José R. Portillo ${ }^{1,4 \bullet^{*}}$, Fernando Soler-Toscano ${ }^{2 \odot}$, Antonio Suárez ${ }^{3,4 \odot}$, José A. Langa ${ }^{3,4 \odot}$ \\ 1 Department of Applied Mathematics I, University of Seville, Spain \\ 2 Department of Philosophy, Logic and Philosophy of Science, University of Seville, \\ Spain \\ 3 Department of Differential Equations and Numerical Analysis, University of Seville, \\ Spain \\ 4 Instituto de Matemáticas de la Universidad de Sevilla Antonio de Castro Brzezicki \\ (2These authors contributed equally to this work. \\ * josera@us.es (JRP)
}

\section{Abstract}

Dynamical systems on graphs allow to describe multiple phenomena from different areas of Science. In particular, many complex systems in Ecology are studied by this approach, for which there exists a huge literature on the dependence of the topology of underlying graphs and their associated dynamics. In this paper we focus on bipartite graphs describing mutualistic antagonistic systems from population dynamics. We study in detail, mathematically and computationally, the zones characterizing different levels of biodiversity. In particular, we investigate the dependence of the region for maximal biodiversity of a system (structural stability) on its connectivity matrix. We analyse the mathematical framework for the study of the structural stability of each stationary point, feasible or not, introducing a global approach to structural stability in mutualistic systems. This property is defined as Global Structural Stability. On the other hand, as network topology does not completely determine robustness of the dynamics on a complex network, we study structural stability as a function of different measures on graphs. A systematic study with synthetic and biological graphs is presented, including mutualistic networks of plants-birds (one from Spain and another from Trinidad and Tobago), plants-pollinators (Japan) and plants-ants (Australia). We compare the relevance of measures of nestedness, fractional matching number, mean centrality and modularity, concluding the importance of latter ones when describing areas of maximal biodiversity. We show that cooperation parameters play the central role for biodiversity and, moreover, the less modular this cooperation is, the more robust its structural stability.

\section{Author summary}

There exists a close relation between the structure of a complex network, described as a graph, and its associated dynamics. Mutualistic networks introduce cooperation links between two groups of species, as plant and pollinators or seed dispersal. The understanding of organizational aspects leading to maximizing biodiversity is one of the more important research areas in Theoretical and Applied Ecology. In this work we introduce a systematic study on different graph measures in order to identify optimal organization for maximal biodiversity (defined as structural stability). Our results 
conclude that, for mutualistic systems, the presence of community cooperation, i.e., cooperation which is highly and more fairly shared among the set of species, is the one optimizing biodiversity. This study opens the door to a fully research plan in order to extend the results to other different real networks and graph characterizations.

\section{Introduction}

Phenomena from Natural and Social Sciences are usually modeled as complex networks for which a dynamic is defined among the nodes $([1-4])$, sometimes associated to dynamical graphs ( $3,5,5]$ ), and where the study of stability is frequently a crucial fact ( [10,11]).

From the keynote paper from Strogatz [9], a lot of research has been added to the study of possible scenarios for the long time dynamics on a complex network with a given topology [12 15], being Population Dynamics [16 19], Economy 20 22] and Neuroscience 2328 some of the areas where this important problem has been intensively treated. Most of these papers infer that the underlined topology of a complex network is somehow associated to the observed dynamics. Indeed, the dependence of the forwards scenarios of a system on the topology of the underlying graph is usually pointed out ( $\left.\begin{array}{|l|l|l|l|l|l|l|l|l|l|l|l|l|l|}13 & 14 & 17 & 19 & 21 & 22 & 26 & 29 & 34\end{array}\right)$. When the dynamics of the system is given by a set of differential equations, its behaviour generically depends on its global attractor ( $35-38]$ ), defined as Informational Structure (IS) (Fig. 9 of Supplementary Material S1) when its geometrical characterization is available ( $[28, \mid 39]$ ). An IS includes not only the information from the topology of the graph (structural network), but other key components that are crucial to understand all possible future scenarios.

On the other hand, in Theoretical Ecology the study of cooperative interactions between groups of plants and pollinators / seed dispersals / ants and how they affect to biodiversity has received an intensive research in the last fifteen years ( 16 19,21]). A mathematical model by a system of differential equations for mutualistic networks in Ecology was introduced in Bastolla et al. 22. Since then, many studies have been focused on this model class, as they provide a precise analysis for a global approach to these complex phenomena. They are represented by bipartite graphs representing two kind of species (classified into two sets, plants and animals) and the cooperative links between the groups (see also $17,19,30,40]$ ). In this paper we focus on $N$-dimensional Lotka-Volterra systems used in Population Dynamics (see, for instance, [41, 42]), but, by the Fundamental Theorem of Dynamical Systems [43], the results of this research can be extended to more general systems of differential equations. We show the dependence of dynamics on the topology of the graph, but, in addition, we claim that this fact it is only part of a more general principle: the dynamics on a graph is globally described by its associated IS, which is different from the structural base graph and whose nature is essentially informational. The IS for these systems is described as an hierarchical set of stationary solutions linked by associated stable and stability manifolds, and informs not only on all the possible future scenarios of the system, but the way they are reached (metastability), the rate of convergence, and the zones describing phase transitions between different structures (bifurcation phenomena).

A mathematical model of differential equations describes the dynamics of nodes on a mutualistic system as follows: suppose $P$ is the total number of plants and $A$ the number of animals. Plants (and animals) are in competition and plants and animals have cooperation links. We introduce the following system of $n=P+A$ differential equations for $S_{p_{i}}$ and $S_{a_{i}}$ describing the species densities populations for the $i$-th species: 


$$
\left\{\begin{aligned}
\frac{d S_{p_{i}}}{d t} & =S_{p_{i}}\left(\alpha_{p_{i}}-\sum_{j=1}^{P} \beta_{p_{i j}} S_{p_{j}}+\sum_{k=1}^{A} \gamma_{p_{i k}} S_{a_{k}}\right) \\
\frac{d S_{a_{i}}}{d t} & =S_{a_{i}}\left(\alpha_{a_{i}}-\sum_{j=1}^{A} \beta_{a_{i j}} S_{a_{j}}+\sum_{k=1}^{P} \gamma_{a_{i k}} S_{p_{k}}\right) \\
S_{p_{i}}(0) & =S_{p_{i 0}} \\
S_{a_{i}}(0) & =S_{a_{i 0}}
\end{aligned}\right.
$$

for each $p_{i}$ for $1 \leq i \leq P$ and $a_{i}$ with $1 \leq i \leq A$. $\alpha_{p_{i}}$ and $\alpha_{a_{i}}$ ( $\alpha_{i}$ in short) are the intrinsic growth rates in the absence of competition and cooperation for plants and animals, respectively, $\beta_{p_{i j}} \geq 0, \beta_{a_{i j}} \geq 0$ denote the competitive interactions and $\gamma_{p_{i j}} \geq 0$ and $\gamma_{a_{i j}} \geq 0$ the mutualistic strengths. Structural Stability focuses in the size of the region for the intrinsic parameters $\alpha_{i}$ to reach optimal (maximal) biodiversity. In 22 (see also $17,19,30,40]$ ) it is studied how the architecture of the network relates to biodiversity. In particular, under some conditions is observed that the more nestedness of the network ( [4] $)$, the more probability for a richer biodiversity; on the other hand, it also depends on other properties of the associated graph, and cannot be considered as the only marker for a higher biodiversity (see, for instance, [45]).

Structural Stability for this model is introduced in [40] as a proper concept unifying the influences of network topology and parameter dependence in the system; it has been used as a proper framework in Theoretical Ecology to analyse robustness of biodiversity in these complex networks ( $[46 \sqrt[48]{48})$. Essentially, structural stability of system (1) measures the region of intrinsic parameters of species for which we get maximal biodiversity. Note that a greater region for structural stability allows for lower values of individual intrinsic growth parameters but preserving a high level of biodiversity, pointing for robustness and resilience of species.

Observe that (1) can be written as a general Lotka-Volterra model for $n$ species as:

$$
\dot{u}_{i}=u_{i}\left(\alpha_{i}+\sum_{j=1}^{n} a_{i j} u_{j}\right), \quad i=1, \ldots, n,
$$

or, equivalently,

$$
\dot{u}=u(\alpha+A u)
$$

with $A=\left(a_{i j}\right)$ the interaction (or adjacency) matrix given by

$$
A=\left[\begin{array}{cc}
B_{1} & \Gamma_{2} \\
\Gamma_{1} & B_{2}
\end{array}\right]_{(P+A) \times(P+A)} .
$$

In this paper we study the structural stability for every possible future scenario of the system, i.e., for the whole set of stationary points (asymptotically stable, semistable or even globally unstable). Following the Linear Complementary Theory (LCP) associated to Lotka-Volterra systems ( $41,42,49]$ ), we introduce a partition of the phase space ( [50]) for which we can estimate the area for which each stationary solution is globally stable by measuring the intersection of its associated cone of biodiversity with the unit $n$-dimensional sphere, defined as Global Structural Stability (see Methods). Moreover, we study in detail the dependence of structural stability on several graph measures characterizing the underlying network described by adjacency matrix $A$ (Results). Our findings allow us to claim that all members cooperation, the one depending on the dispersion of degree distribution on the graph, seems the better configuration (among other graph markers as nestedness or modularity, for instance) for optimization of maximal biodiversity in mutualistic systems. Moreover, to equal degree 
distribution, modularity also seems to play an important role for biodiversity. After a deep computational analysis on nine-node bipartite graphs, we also study several networks related to real ecosystems of mutualistic type to conclude and illustrate the scope of our theoretical results.

\section{Materials and Methods}

\section{Global Structural Stability}

The study of the size of the region for intrinsic growth parameters (in our case the $\alpha_{i}$ parameters) for which a system reaches its optimal biodiversity (all the species present) is defined as Structural Stability in 40. This is a crucial fact for the study of the robustness of biodiversity in an ecosystem, as it characterizes the borders for intrinsic growth to get maximal biodiversity. However, specially in high dimensional systems, a stationary point with all its components strictly positive either does not exist, or, if this is the case, there also exists a big set of semistable stationary points. The presence of these stationary points are crucial for the description of the transient behaviour and metastability properties of the system, so that to neglect its study could lead to wrong conclusions. For instance, the transition to one globally asymptotically stationary point to other by a bifurcation parameter is usually described as a sudden phenomena, but, as we will try to explain, it is totally understandable by a careful study of the parameter region of stability for each stationary point and the way they intersect. Moreover, the ways to reach a particular stationary solution are multiple, depending of the different (informational) landscapes ( 39 ) described in detail by its semistable solutions. Indeed, under the hypotheses of $A$ in (3) to be Lyapunov-stable, it is known that there exits a unique global asymptotically stable stationary point ( [41]). But there also exists a huge set (at most $2^{n}$ ) of actual stationary points which are determining the transient dynamics, and describing the closeness to phase transitions between different scenarios of biodiversity. In this section we introduce a full study of the structural stability of each stationary point in the system, independently of their stability properties. This is referred as Global Structural Stability. For (3), the zero solution is globally unstable ( $n$ directions of instability), each stationary $u_{j}^{*}$ point belongs to an informational level $E_{i}$ and possesses stable and unstable directions, and there exists just one stationary feasible point $u *$ in the lower level which is globally stable (see Fig 10 in S1).

To illustrate the description of global structural stability, consider a two-dimensional cooperative system given by

$$
\left\{\begin{array}{c}
\dot{u}_{1}=u_{1}\left(\alpha_{1}-u_{1}+a u_{2}\right) \\
\dot{u}_{2}=u_{2}\left(\alpha_{2}-u_{2}+b u_{1}\right)
\end{array}\right.
$$

with $\alpha_{i} \in \mathbb{R}$ and $a, b>0$. For a fixed network of connections in the system (given by values of the $a$ and $b$ parameters), the intrinsic growth rate of each species plays a crucial role. Indeed, a convex cone of $\alpha$ parameters in (4) is associated to these stationary points, and all of these convex cones form a partition of $\mathbb{R}^{2}$ (see $[50]$ ), i.e., each cone has a nonvoid interior, the union of all cones is $\mathbb{R}^{2}$ and each pair of the interior of cones is disjoint (see Fig 1).

This means that a given vector $\alpha$ of (4) belongs either to just the interior of one cone (determining the feasible stationary point, and so the future biodiversity of the system) or to the intersection of cones, made by rich manifolds showing a phase transition and a high sensibility to bifurcation scenarios in biodiversity.

In Fig 2 we show the different cones for a three dimensional Lotka-Volterra system. Note, once more, that the union of cones form a partition of $\mathbb{R}^{3}$. 

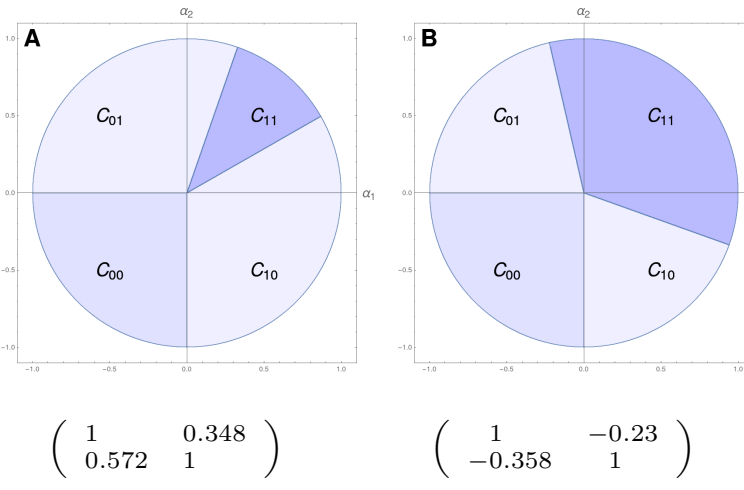

\begin{tabular}{lcc} 
Cone & $\mathbf{A}$ & $\mathbf{B}$ \\
\hline$C_{00}$ & 0.250 & 0.250 \\
$C_{01}$ & 0.303 & 0.214 \\
$C_{10}$ & 0.333 & 0.195 \\
$C_{11}$ & 0.114 & 0.341
\end{tabular}

Fig 1. Description of cones for alpha parameters associated to 4 with $2 \times 2$ matrices as indicated. A. competitive case. B. cooperative case. Note that there are four regions $C_{i j}, i, j=0,1$, each for the four possible stationary points. If $\alpha \in C_{i j}$, the globally asymptotically stable point $u^{*}$ for (4) has the positive components pointed by $i j$, i.e., $\alpha \in C_{11}$ means that in $u_{1}^{*}, u_{2}^{*}>0$. Moreover, note that borders of each cone are bifurcation submanifolds, in the sense that a sudden attractor bifurcation occurs when passing through this border. Moreover, inside each cone, there exists interesting zones marking different attractor structures with the same globally asymptotically stable solution.

\section{A}

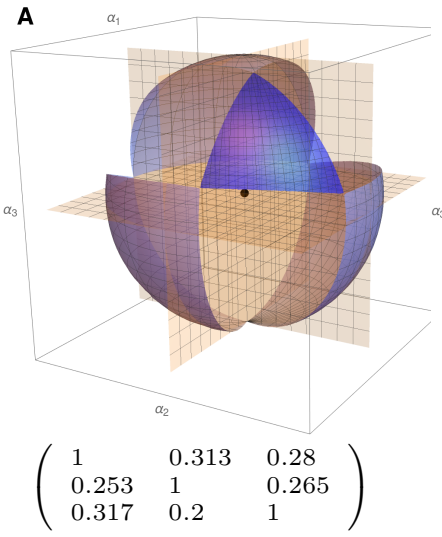

B

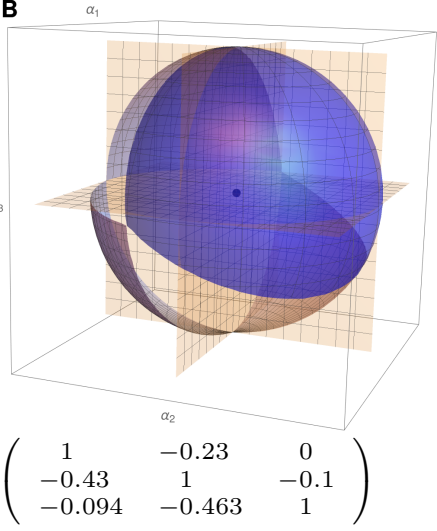

\begin{tabular}{lcc} 
Cone & $\mathbf{A}$ & $\mathbf{B}$ \\
\hline$C_{000}$ & 0.125 & 0.125 \\
$C_{001}$ & 0.173 & 0.117 \\
$C_{010}$ & 0.169 & 0.080 \\
$C_{011}$ & 0.116 & 0.139 \\
$C_{100}$ & 0.175 & 0.088 \\
$C_{101}$ & 0.099 & 0.089 \\
$C_{110}$ & 0.102 & 0.101 \\
$C_{111}$ & 0.040 & 0.260
\end{tabular}

Fig 2. Two representation of the eight cones describing global structural stability. A. a 3D competitive LV system, with the cone of maximal biodiversity (in blue). B. a 3D cooperative LV system, with a bigger cone of maximal biodiversity (in blue), pointing the key role of cooperation in biodiversity. 
We will refer to Global Structural Stability as the study of the properties of regions describing the different possible scenarios of biodiversity in an ecological system. The size of the cone-partition of the phase space and its dependence on the structural network of connection between species suppose core subjects in this analysis.

The study of the structural stability for all possible asymptotic realization informs, in a holistic way, on the robustness of a particular future scenario related to other possible ones.

\section{Network characterization and structural stability}

Antagonism/competition ( $\beta_{i j}$ parameters) is established between all pairs of nodes in the same set and mutualism/cooperation $\left(\gamma_{i j}\right)$ between nodes of different sets. The competition relations are constant in most experiments (random in some cases), only the cooperation part changing across all graphs. If we only look at the cooperation relationships, the graphs are bipartite. We will call these graphs as $\gamma$-bipartite and they have a similar structure to the example in Fig 11 where the weights of edges connecting different sides correspond to cooperation $\gamma_{i j}$ parameters and inside each group of nodes (left and right) competition $\beta_{i j}$ parameters are fixed. This structure is familiar in the study of mutualistic ecological networks $18,19,51$, like plants-pollinators, plants-seed dispersals or plants-ants relationships. As explained above, $\beta_{i j}=\beta_{j i}$ and $\gamma_{i j}=\gamma_{j i}$. Also, $0<\beta_{i j}, \gamma_{i j}<1$ and $\sum_{j}\left(\beta_{i j}+\gamma_{i j}\right)<1$ for each $i \in\{1, \cdots, n\}$.

Given a system with a fixed connectivity matrix (parameters $\beta_{i j}$ and $\gamma_{i j}$ ), it is possible to determine if $\left(\alpha_{1}, \cdots, \alpha_{n}\right)$ is in the maximum biodiversity cone.

Given a connectivity matrix $M$ (like the example in Fig 11) we estimate structural stability by using a Monte Carlo method. The process is summarised in the following python code:

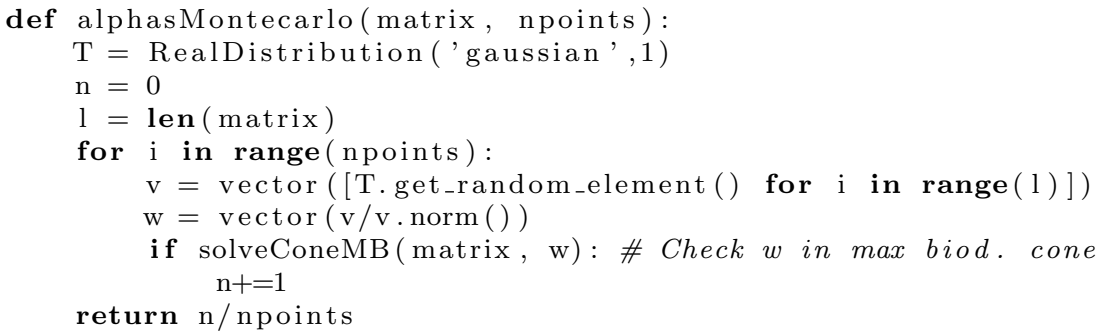

The input of the function is the connectivity matrix (with the structure of $M$ in (6) and npoints, the number of random points to be generated by the Monte Carlo method (the greater is npoints, the better is the returned estimation; in our case the number of points is $\left.4 \times 10^{6}\right)$. The procedure consists on generating npoints random points on the surface of the sphere of radius 1 in $\mathbb{R}^{N}$ and check whether they are on the maximum biodiversity cone by (7). To ensure that the points are uniformly generated on the surface of the sphere, vector $\mathrm{v}$ is generated (line 6 ) by obtaining random values of the standard normal distribution ( $\mathrm{T}$, line 2 ). Vector $\mathrm{w}$ is obtained dividing $\mathrm{v}$ by its norm (line 7), so $\mathrm{w}$ is a point in the sphere of radius 1. If w verifies Eq. (7) (this is what line 8 checks by calling solveConeMB (matrix, w)), then 1 is added to the counter $\mathrm{n}$ of points in the maximum biodiversity cone. The output of the function is $n / n p o i n t s$, which approximates the structural stability of the system represented by matrix, that is, the proportion of the sphere of radius 1 intersecting the maximum biodiversity cone. 


\section{Results}

\section{Graph characterization and structural stability}

We have explored the relation between structural stability and several graph measures. We focus on connected graphs representing networks with nodes divided into two sets of size 4 and 5 . Note that the adjacency matrix of our graphs is matrix $A$ in (3).

Three kinds of experiments are presented (Tables 1 and 2). Spearman correlation coefficient $\rho$ is used. It supposes a non-parametric measure on the correlation between two (discrete or continuous) random variables (see $[52$ ). To compute $\rho$, data are ordered and replaced by their respective order. In the first set of experiments, all 558 $\gamma$-bipartite connected graphs of $4+5$ nodes have been generated with nauty [53]. These graphs are the basis of the first four studies. Study A is done by weighting graph edges such that all $\beta_{i j}=0.1$ and $\gamma_{i j} \geq 0.05$ maximise the Fractional Perfect Matching of each graph (see below for the presentation of the graph measures). Study B sets for each $\gamma$-bipartite graph all $\beta_{i j}$ and $\gamma_{i j}$ to 0.125 . Study $\mathrm{C}$ assigns random values to all non-zero $\beta_{i j}$ and $\gamma_{i j}$ parameters, ensuring that the sum of the weights of the edges adjacent to each node (node degree) is less than one. Study D sets all $\beta_{i j}=0.05$ and $\gamma_{i j}$ are random variables (bounding the sums to 1 like in $\mathrm{C}$ ).

The second kind of experiments uses 500 instances of the complete $\gamma$-bipartite graph $K_{4,5}$. Edges are weighted in different ways. Experiment AZ is based, as experiment A, in Fractional Perfect Matching (FPM): $\gamma_{i j}$ parameters in the 500 complete $\gamma$-bipartite graphs are optimised to obtain the maximum average degree of the graph such that the sum of the edge weights adjacent to each node is less than one. This maximum average degree is by definition, the FPM divided by the number of nodes, 8.4/9 in this case. These 500 graphs are obtained by requiring to each node $n_{i}$ a minimal value for $\sum_{j} \gamma_{j i}$ (connections from all nodes $n_{j}$ in the group opposite to $n_{i}$ ), called Minimal Degree Restriction (MDR), ranging from $0.17 / 500$ to 0.17 (where all $\gamma_{i j}$ are about equal), one different value per graph. All $\beta_{i j}=0.05$. Experiment AY follows this idea, now optimising $\gamma_{i j}$ values (also in 500 complete graphs) such that the average degree of the graph is equal to $5 / 9$ for all graphs. BZ studies $\gamma$-bipartite complete graphs with $\beta_{i j}=0.05$ and all $\gamma_{i j}$ having a constant value, varying linearly from $0.17 / 500$ to 0.17 . CZ compared $500 \gamma$-bipartite complete graphs with all $\beta_{i j}$ and $\gamma_{i j}$ with random values (bounding edge degree to 1). Finally, DZ compares 500 instances of the complete $\gamma$-bipartite graph with all $\beta_{i j}=0.05$ and random $\gamma_{i j}$ values like in $\mathrm{D}$.

Table 1. Summary of the first two groups of experiments with graphs of $4+5$ nodes. The first group (left column) studies all $558 \gamma$-bipartite graphs of $4+5$ nodes, by setting $\gamma_{i j}$ and $\beta_{i j}$ in different ways. The second group (right column) focuses on the complete graph of $4+5$ nodes (all $\beta_{i j}, \gamma_{i j}>0$ ), with 500 copies of the graph with different connectivity values. Structural stability is approximated with $4 \times 10^{6}$ Monte Carlo points for each connectivity matrix in the experiments.

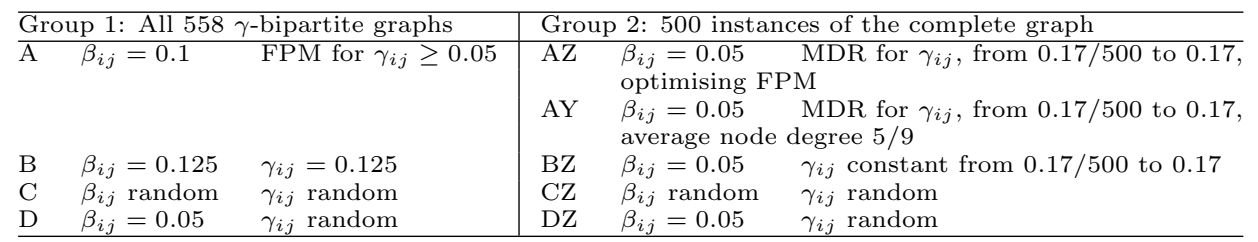

These first two studies aim at a comprehensive exploration of the relationship between structural stability and several network measures. This is why the construction of the graph sets is intended to cover as much of the search space (connected $4+5$ node 
graphs) as possible. To analyse what happens in real biological networks we have done similar experiments using data from mutualistic networks previously introduced in the literature. We have selected the following networks:

1. Snow65_14 [54], a mutualistic network of 65 plants and 14 fruit-eating birds acting as seed-dispersers in Trinidad (Trinidad and Tobago).

2. M_PL_054 [55], a mutualistic network of 431 plants and 113 pollinators in the campus of Kyoto University (Japan). We have extracted the 30 plants and 60 insects with more interaction.

3. M_SD_20 [56, a mutualistic network of 25 plants and 33 birds acting as seed-dispersers in S. Cazorla (Andalusia, Spain).

4. M_PA_004 [57, a cooperation network between 41 ants and 48 plants in Cape Tribulation (Far North Queensland, Australia).

The first network has been obtained from the Interaction Web DataBase (https://iwdb.nceas.ucsb.edu/) and the other three from the Web of Life (http://www.web-of-life.es/).

For each of these networks we have randomly extracted 500 different subnetworks of order $4+5$, requiring that the resulting underlying bipartite graphs are connected in order to compare the results with previous experiments. The cooperative interactions were then normalised to obtain the $\gamma_{i j}$ coefficients between 0 and 1 such that the sum of $\gamma_{i j}$ is less to 0.8 for every row and column. This normalisation has been done in two different ways. The normalisation in the .AX experiments is similar to that of the A, $\mathrm{AZ}$ and AY experiments, in which all networks have the same total sum of $\gamma_{i j}$. In the other kind of normalisation (for .CX and .DX), all the $\gamma_{i j}$ have been divided by the maximum sum of rows and columns of all the (randomly selected) subnetworks. With respect to the antagonistic $\beta_{i j}$ coefficients (modelling competition between species) they were given a constant value of 0.02 in the .AX normalisation (where the $\gamma_{i j}$ coefficients are smaller). In the other normalisations they have been assigned values in two different ways, so that the experiments can be compared with previous theoretical experiments. In .CX, $\beta_{i j}$ have random values between 0 and 0.05 , similar to experiments $\mathrm{C}$ and $\mathrm{CZ}$. In .DX, fixed $\beta_{i j}=0.05$ are used, as in experiments D and DZ. All choices of $\beta_{i j}$ guarantee that for all graphs the sum of the elements of each row and column is less than 1 . These operations have been summarised in Table 2 .

Table 2. Summary of the experiments with 500 randomly selected graphs of $4+5$ nodes extracted from biological networks (Snow65_14 [54], M_PL_054 [55], M_SD_20 56] and M_PA_004 [57]). Structural stability is approximated with $4 \times 10^{6}$ Monte Carlo points for each of the 500 subnetworks.

\begin{tabular}{l|ll}
\hline AX & $\beta_{i j}=0.02$ & $\Sigma \gamma_{i j}$ constant \\
.CX & $\beta_{i j}$ random & $\gamma_{i j}$ normalized globally \\
.DX & $\beta_{i j}=0.05$ & $\gamma_{i j}$ normalized globally \\
\hline
\end{tabular}

In all these graph sets we have studied the correlation between structural stability and several graph measures. Our aim is to get insight into the criteria producing connectivity matrices with higher structural stability. Degree centrality and optimal modularity are studied in all experiments. In the first group of experiments (Table 1), nestedness and fractional matching number are also considered. Tables 3 (first group of experiments), 4 (second group) and 5 (biological mutualistic networks) show the Spearman correlation coefficient between these parameters and structural stability, estimated by using $4 \times 10^{6}$ Monte Carlo points in each connectivity matrix. 
Table 3. Spearman correlation coefficients between structural stability and several graph measures for the experiments in the first group (see the left column of Table 1 for the value of $\gamma_{i j}$ and $\beta_{i j}$ parameters) considering all $\gamma$-bipartite graphs with $4+5$ nodes.

\begin{tabular}{lrrrr}
\hline Parameter & A & B & C & D \\
\hline Degree (mean) & 0.757 & $\mathbf{0 . 9 8 9}$ & -0.165 & $\mathbf{0 . 9 7 0}$ \\
$\sum \gamma_{i j}-\sum \beta_{i j}$ & - & - & $\mathbf{0 . 9 8 5}$ & - \\
Fractional matching number & 0.757 & 0.416 & 0.308 & 0.470 \\
Optimal modularity & -0.726 & -0.743 & -0.461 & -0.441 \\
Nestedness & 0.111 & 0.382 & 0.263 & 0.072 \\
\hline
\end{tabular}

Table 4. Spearman correlation coefficients between structural stability and several graph measures for the experiments in the second group considering 500 different instances (see the right column of Table 1 for the way in which $\gamma_{i j}$ and $\beta_{i j}$ change) of the complete $\gamma$-bipartite graphs of $4+5$ nodes. Missing correlations are because in some cases all graphs have the same mean degree and MDR is not used in some others.

\begin{tabular}{lrrrrr}
\hline Parameter & AZ & AY & BZ & CZ & DZ \\
\hline Degree $($ mean $)$ & - & - & $\mathbf{0 . 9 9 9 9 8}$ & 0.011 & $\mathbf{0 . 9 9 5}$ \\
$\sum \gamma_{i j}-\sum \beta_{i j}$ & $\mathbf{0 . 9 9 9 7}$ & $\mathbf{0 . 9 9 9 9 8}$ & - & $\mathbf{0 . 9 8 7}$ & - \\
Optimal modularity & $\mathbf{- 0 . 9 6 9}$ & $\mathbf{- 0 . 9 9 7}$ & -0.732 & -0.017 & 0.024 \\
\hline
\end{tabular}

\section{Centrality measures}

Centrality is a measure that determines the relative importance of a node within a network $[58,59]$. Being able to recognise the centrality of a node can help to determine, for example, the impact of a person involved in a social network, the relevance of a room in a building represented in spatial syntax, the importance of a road in an urban network, or the essential components of a computer network.

Centrality is not an intrinsic attribute of the nodes or actors in a network, such as temperature, monetary income, etc., but a structural attribute, that is, an assigned value which strictly depends on its location in the network. Centrality measures the contribution of a node according to its location in the network, regardless of whether its importance, influence, relevance or prominence is being assessed.

Degree centrality corresponds to the number of links that a node has with the others 60. It provides the clearest relationship with structural stability in most cases. As degree centrality is a measure of the nodes, we use for each graph the mean degree of its nodes. This correlation is specially high in experiments B and D (Table 3), BZ and DZ (Table 4), and in all the .DX experiments with biological networks: Snow_14DX , M_PL_054DX, M_SD_020DX and M_PA_004DX (Table 5). In all these cases the Spearman correlation is greater than 0.9. See Fig 3 for a graphical representation of these results.

Observe that in some cases Spearman correlation between structural stability and degree centrality is not significant (experiments $\mathrm{C}$ and $\mathrm{CZ}$ ). But it again appears if instead of considering the mean (or, equivalently, the sum) of $\beta_{i j}$ and $\gamma_{i j}$ $\left(\sum \beta_{i j}+\sum \gamma_{i j}\right)$ we look at the difference $\left(\sum \gamma_{i j}-\sum \beta_{i j}\right)$. See Tables 3 and 4 and Fig 4 .

Similarly, in .CX experiments with biological networks, where random $\beta_{i j}$ are used, the correlation also grows by studying the difference instead of the sum (Fig. 5 and Table 5). 

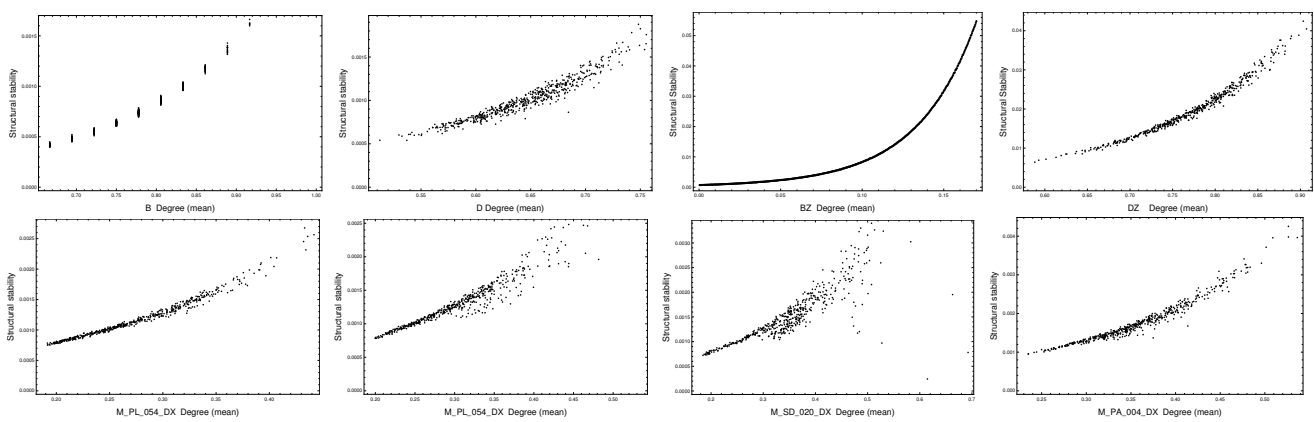

Fig 3. Degree centrality and structural stability. Top (left to right): B, D, BZ, DZ. Bottom (left to right): Snow_14DX , M_PL_054DX, M_SD_020DX, M_PA_004DX. Spearman correlation coefficient is greater than 0.89 in all these cases (Tables 3 , 4 and 5). In these graphs, degree centrality grows with the sum of the $\gamma_{i j}$ parameters (as $\beta_{i j}$ are equal in all graphs), showing that the more cooperation, the higher structural stability.
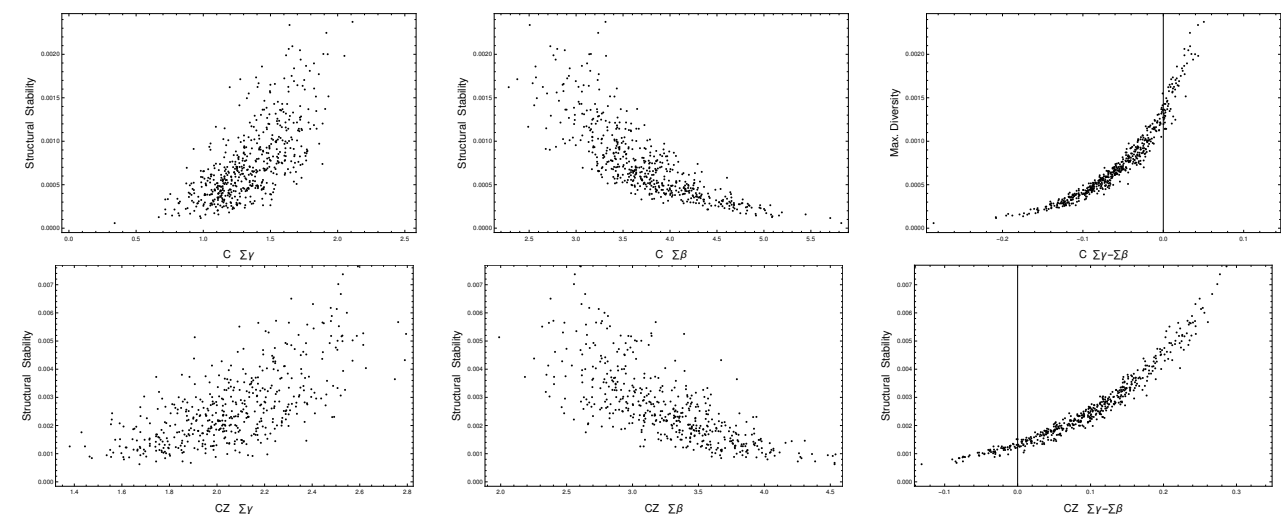

Fig 4. Sum of cooperation $\sum \gamma_{i j}$ (left column), competition $\sum \beta_{i j}$ (centre) and their difference $\sum \gamma_{i j}-\sum \beta_{i j}$ (right) versus structural stability in graphs with random edge weight: $\mathrm{C}$ (top row) and CZ (bottom). Correlations for $\sum \gamma_{i j}$ and $\sum \beta_{i j}$ are shown in Table 6. Correlations for $\sum \gamma_{i j}-\sum \beta_{i j}$ are shown in Tables 3 and 4 . Results indicate that the relationship of structural stability is direct to the sum of the cooperation coefficients and inverse to the sum of the competition ones. As (see Table 1 ) all $\gamma_{i j}$ and $\beta_{i j}$ are random, the best correlations are obtained for $\sum \gamma_{i j}-\sum \beta_{i j}$, above 0.98 in both cases.
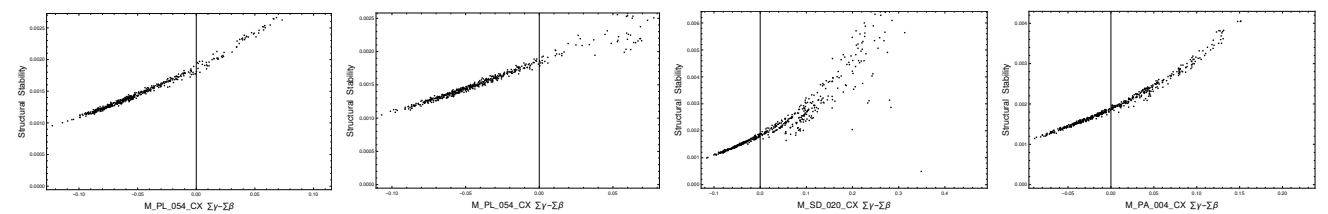

Fig 5. Diference $\sum \gamma_{i j}-\sum \beta_{i j}$ versus structural stability in biological networks with random competition coefficients. From left to right: Snow65_14CX, M_PL_054CX,

M_SD_020CX and M_PA_004CX. Correlations are shown in Table 5 Results indicate that structural stability possesses a direct relation with the sum of cooperative interactions and an inverse relation with the strength of competition. 
Table 5. Spearman correlation coefficients between structural stability and several graph measures for the experiments with biological networks, considering 500 $\gamma$-bipartite graphs of $4+5$ nodes given from randomly selected subnetworks of Snow65_14 [54 (plants-dispersers), M_PL_054 55] (plants-pollinators), M_SD_020 56 (plants-dispersers) and M_PA_004 [57] (plants-ants). See Table 2 for the $\gamma_{i j}$ and $\beta_{i j}$ normalisation. Missing correlations are because all graphs in .AX experiments have the same mean degree and in .DX experiments given that $\beta_{i j}$ are constant, the orders given by $\sum \gamma_{i j}-\sum \beta_{i j}$ and the mean degree are the same.

\begin{tabular}{lrrr}
\hline Parameter / Network &. AX & .CX & .DX \\
\hline Degree (mean) & & & \\
Snow65_14 & - & 0.535 & $\mathbf{0 . 9 9 5}$ \\
M_PL_054 & - & 0.571 & $\mathbf{0 . 9 6 9}$ \\
M_SD_020 & - & $\mathbf{0 . 9 0 3}$ & $\mathbf{0 . 9 1 6}$ \\
M_PA_004 & - & 0.828 & $\mathbf{0 . 9 8 1}$ \\
\hline$\sum \gamma_{i j}-\sum \beta_{i j}$ & & & \\
Snow65_14 & - & $\mathbf{0 . 9 9 5}$ & - \\
M_PL_054 & - & $\mathbf{0 . 9 9 4}$ & - \\
M_SD_020 & - & $\mathbf{0 . 9 7 3}$ & - \\
M_PA_004 & - & $\mathbf{0 . 9 7 2}$ & - \\
\hline Optimal modularity & & & \\
Snow65_14 & -0.503 & -0.671 & -0.540 \\
M_PL_054 & -0.511 & -0.296 & -0.056 \\
M_SD_020 & -0.657 & -0.557 & -0.660 \\
M_PA_004 & -0.301 & -0.340 & -0.162 \\
\hline
\end{tabular}

Degree centrality (and sum of $\beta_{i j}$ and $\gamma_{i j}$ parameters) is constant in experiments AZ and AY, so Spearman correlations cannot be calculated. But in these cases

$\sum \gamma_{i j}-\sum \beta_{i j}$ has also a high correlation with structural stability (Table 4 and Fig 6 ).

The design of the .AX experiments with biological networks produces constant sums of both $\gamma_{i j}$ and $\beta_{i j}$ parameters, so that the study of mean degree does not make sense in this case.

These results suggest that structural stability (ability to produce maximum biological diversity) is related to degree centrality or, equivalently, to the sum of node degrees. But the correlation is even stronger with just the cooperation coefficients $\gamma_{i j}$, when the competition coefficients $\beta_{i j}$ are constant across all graphs in the experiments. Correlations for B, D, BZ, DZ and all .DX experiments in biological networks are always above 0.9 .

To test this claim, we have calculated for C, CZ studies (with all $\beta_{i j}$ and $\gamma_{i j}$ random) the Spearman correlation between structural stability and $\sum \gamma_{i j}$ alone, and the same for $\sum \beta_{i j}$. Results are shown in Table 6 and Fig 4 , confirming that the relationship of structural stability (maximum biological diversity) is direct to the sum of the cooperation coefficients and inverse to the sum of the competition ones. In the .CX experiments with biological networks, where all $\beta_{i j}$ are also random, the structural stability is very strong too. This is a relevant result because it shows that the more cooperation (and less competition) between the nodes within the network, the grater structural stability is produced. Other measures discussed below focus on how the distribution of the cooperation affects structural stability. 
Table 6. Spearman correlation coefficients between structural stability and sums of cooperation $\left(\sum \gamma_{i j}\right)$ and competition $\left(\sum \beta_{i j}\right)$ parameters in experiments $\mathrm{C}$ and $\mathrm{CZ}$. Correlations are not very strong because the connectivity parameters in these experiments are random (Table 1), so both sums change independently across all the considered graphs. Observe that the sum of just one type of parameter (cooperation or competition) misses half of the relevant information. Correlation is above 0.98 by considering $\sum \gamma_{i j}-\sum \beta_{i j}$ (Fig 4 and Tables 3 and 4 .

\begin{tabular}{lrr}
\hline Parameter & $\mathrm{C}$ & $\mathrm{CZ}$ \\
\hline$\sum \gamma_{i j}$ & 0.714 & 0.678 \\
$\sum \beta_{i j}$ & -0.871 & -0.787 \\
\hline
\end{tabular}
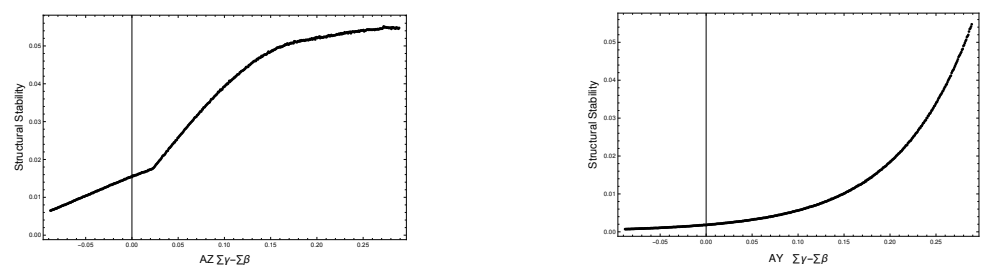

Fig 6. Diference $\sum \gamma_{i j}-\sum \beta_{i j}$ and structural stability in experiments AZ (left) and AY (right). Graphs in these experiments are built using FPM and MDR (Table 1). Spearman correlation coefficients are both above 0.99. Results are similar to those in Fig 4, showing that the direct (inverse) relation of structural stability with cooperation (competition) parameters is robust enough to hold both for random and (FPM/MDR) optimised graphs.

\section{Modularity}

Modularity measures the division of a network into communities (groups, clusters or modules) 61. Its value (between $-1 / 2$ and 1 ) is relative to a partition of the graph nodes into different communities. It is defined as the fraction of the edges within the communities minus the expected fraction if edges were set at random. The maximum value for a graph (considering all possible partitions) is called optimal modularity. Networks with high optimal modularity (close to 1) have high connectivity between nodes within the same community and low connectivity between different communities. Modularity is 1 just in case that communities are complete graphs with no links between them. Some biological networks have high degrees of optimal modularity 25]. Graphs with a very specific structure (grids or similar) have negative optimal modularity.

We have used the C library iGraph 62 which implements the Newman 61 algorithm to compute optimal modularity. Spearman correlation is strong in experiments AZ and AY, in which the sum of the node degrees (degree centrality) is constant (Fig 7). In A, B and BZ, whose graphs are built in such ways that produce a linear growth in total degrees, the correlation is also relevant. As Tables 3 and 4 show, there is no significant correlation in other experiments.

Results in most experiments with biological networks (Snow_14AX, M_PL_054AX, M_SD_020AX, Snow_14CX, M_SD_020CX, Snow_14DX and M_SD_020DX) show a slightly relevant correlation (less than -0.5 ). See Table 5 and Fig 8 . 

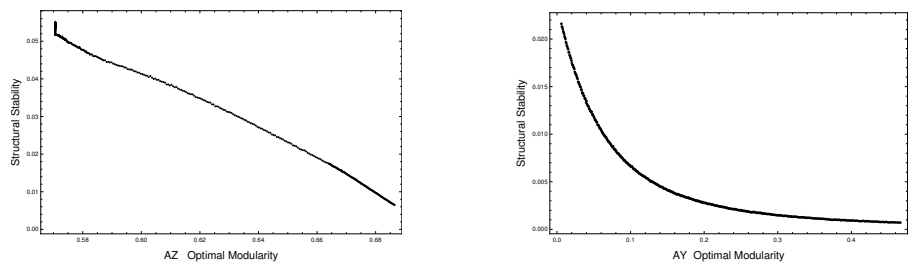

Fig 7. Optimal modularity and structural stability in experiments AZ (left) and AY (right). In both cases the Spearman correlation coefficient if below -0.98. It indicates that the formation of strongly interrelated communities within a biological system has a negative influence on maximum biological diversity. Given a fixed value of $\sum \gamma_{i j}$, the less modular are the $\gamma_{i j}$, the higher structural stability is produced.
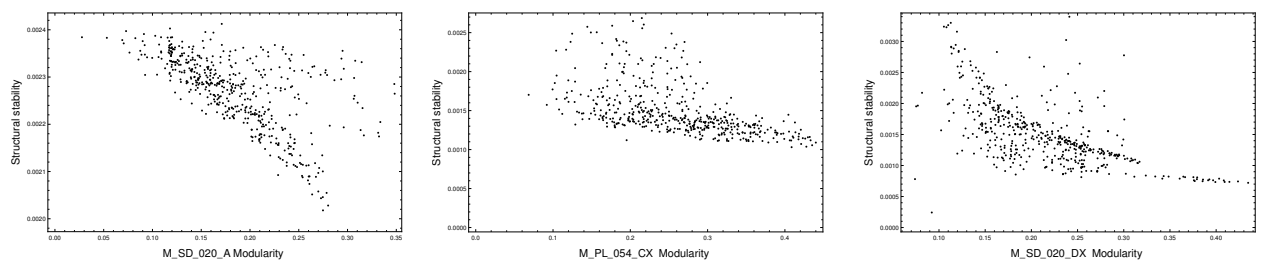

Fig 8. Optimal modularity and structural stability in experiments M_SD_020AX (left) Snow_14CX (center) and M_SD_020DX (right).

\section{Fractional perfect matching}

To finish the experiments about graph parameters, we have studied two more variables: fractional matching number and nestedness. Both parameters are a function of the topological structure of the graph itself and not of the specific $\gamma$ and $\beta$, so they can only be studied in the first group of experiments.

One of the restrictions for the connectivity matrices if that the node degree is always lower than 1. By fixing $\beta_{i j}$ (what is done in all experiments except $\mathrm{C}$ and $\mathrm{CZ}$ ), this is equivalent to require that the sum of the weights of the incident edges at each node is lower to some constant value. It is reasonable to study what happens when the weights corresponding to $\gamma$ values are maximised. This problem is known as fractional perfect matching (FPM) 63 64. Given a graph $G$, a fractional matching is a function $f$ that assigns to each edge $e \in E(G)$ a number in $[0,1]$ so that for each node $v$, $\sum_{e \in E_{v}} f(e) \leq 1$ where $E_{v} \subseteq E(G)$ is the set of edges incident to $v$. The fractional matching number $\mu_{f}(G)$ of $G$ is the supremum of $\sum_{e \in E(G)} f(e)$ over all fractional matchings $f$. The fractional matching number can be computed using linear programming. The matrix of coefficients of this program is the incidence matrix $M(G)$ of $G$. We have used this property to calculate with a simple Mathematica program the fractional matching number of all $\gamma$-bipartite graphs with $4+5$ nodes.

The correlation with structural stability is low for experiments of the first group (Table 3). In the case of experiment A, the correlation is identical to the one for mean degree because the construction of the graphs produce the same value for both measures. In the second set of experiments FPM cannot be studied because it is constant in complete graphs. However, we can look at experiments AZ and AY, whose graphs are built by using FPM, requiring that all $\gamma_{i j} \geq$ MDR (see Table 1). We have studied the correlation between MDR and structural stability in experiments AZ and AY. Spearman correlation coefficients are identical to those obtained for the correlation between $\sum \gamma_{i j}-\sum \beta_{i j}$ and structural stability (see Table 4 and Fig 6). This identity is due to the graph construction using FPM and MDR (the greater MDR, the higher $\sum \gamma_{i j}-\sum \beta_{i j}$ ) and because the Spearman coefficient does not depend on the exact values of the 
compared variables but the relative order of the elements in the respective ranks. So the relation shown in Fig 4 between structural diversity and $\sum \gamma_{i j}-\sum \beta_{i j}$ (direct relation for cooperation, inverse for competition) for random graphs also holds in these graphs which are optimised by FPM and MDR.

\section{Nestedness}

Nestedness is used in Ecology to measure a kind of organization of an ecological system 65. The species are ordered from generalist to specialist. A network is nested when its specialists interact with subsets of the species that interact with the generalists. An ecological conjecture is that the more nested a network is, the more robust is to perturbations $[17,19,30,66,68$.

We have used R 69$]$ library FALCON 70 to get the nestedness value of the bipartite graphs in the first set of experiments. The Spearman correlation with structural stability is very low in all cases (Table 3). Nestedness is not studied in the second set of experiments (as they are weighted complete graphs). Nestedness is a function of the graph structure. Thus, the calculation has been done over the associated non-weighted graph. There are attempts to define weighted versions of nestedness (see 71]) but there is an agreement that weighted nestedness does not measure the same network properties. Thus, our results do not show any evidence for the value of nestedness in order to increase structural stability, which is in line with the necessity of understanding nestedness depending on other conditions to show its value as biomarker in this kind of networks (see, for instance, the discussion in 45]).

\section{Discussion}

The level of the interdependence between structure and dynamics on complex networks is not always clear; sometimes it seems that this relation induces determination, and usually just correlation in most cases. In this paper we conclude that dynamics, although closely related, is not determined by the topology of the network. We have introduced the object (Informational Structures) that could explain, under some hypotheses, the nature of this relation and, what is more important, the object from which we could also understand all the possible future scenarios of phenomena described by a dynamical system.

To study the dependence of the topology and its associated dynamics we have introduced an $n$-dimensional Lotka-Volterra system of differential equations. The adjacency matrix associated to this system describes the underlying structural graph. Cooperative type Lotka-Volterra systems suppose a class in which, by the nature of the positive input on existing links among nodes, maximizes the influence of network topology on the dynamics. Indeed, it is the class of systems from which the richness on the number of existing connections has a direct consequence on the plurality (diversity) of future scenarios.

In general, to study the long time behaviour of a dynamical system, it is a common way to analyse its stationary points and associated stability. An IS contains this information as a whole, all the possible scenarios at the same time, their stability, their robustness, and, crucially, the way they are joined. All this information are usually belittled in many research contributions. ISs are described in our Lotka-Volterra system with precision, so that allowing for further studies on robustness, bifurcation phenomena or metastability of solutions, in which the role of the associated informational field may be crucial $28,39,72,74]$.

The Fundamental Theorem of Dynamical Systems 43,75 allows for the same kind of conclusions if other nonlinearities are considered in our complex network. That is, if 
we introduce other vector fields associated to the differential equations, we should also expect full determination from the description of the associated informational structures.

The concept of structural stability is used in 22], and defined as in the present paper in 40] (see also [6]). In this work we have introduced a global framework to study the structural stability for every possible stationary point of a mutualistic system. It is very important to determine the robustness of each asymptotic regime, measured by the region that associated parameters reach. In this sense, we have introduced an $\mathbb{R}^{n}$-partition for the $\alpha$-parameters describing the different convex regions for each stationary solution, which is globally asymptotically stable in these regions. To our knowledge, this is the first time structural stability is used to study all the possible future scenarios, and not only to determine maximal biodiversity. We are aware we have not taken all the important information from the existence of an informational structure. Indeed, there are many possible configurations possessing the same global asymptotic stable stationary solution (see for instance Figure 10 in which the set of semistable stationary points above the last asymptotically stable one could be very different), and would deserve further research.

Our results also suggest that optimal modularity has a negative impact on biological diversity (structural stability), but in a smoother way that the combination of total degree of cooperation $\gamma_{i j}$ (positive influence) and competition/antagonism $\beta_{i j}$ (negative influence). That is, the formation of strongly interrelated communities within a biological system has a negative influence on maximum biological diversity. More conclusive results on modularity may require more extensive studies with larger networks, as graphs of size $4+5$ are too small to observe complex modularity phenomena.

In a seminal work, Bascompte and collaborators 30] showed that real mutualistic networks are significantly nested. They also study how the so-defined nestedness of empirical mutualistic networks is correlated with the biodiversity of the corresponding ecosystems 22]: the global species competition is significantly reduced by developing a nested network architecture and this entails a larger biodiversity. However, the importance of nestedness for an increasing of biodiversity is not so straightforward, and the discussion is still active, essentially depending on the set of equations, the kind of interactions or the weak or strong cooperation regime in the mutualistic case (see, for instance, $77-80]$ ). Our results show no direct relation between nestedness and structural stability. Note that we move in the weak cooperation regime (see [22 45]) and we are relating nestedness to the size of the set of growth parameters for which maximal biodiversity holds, so that there is no contradiction with previous works in literature, just adding new results in this challenging discussion. Finally, an interesting work on the relation between nestedness and modularity appears in [51]. Observe that, in our case, we cannot conclude this kind of correlations because we are calculating optimal modularity on weighted graphs, while only simple graphs are used in 51. Moreover, the graph order in 51] is much higher, although, due a lower order in our case, we are able to do an exhaustive study of all possible networks.

\section{Software}

Software code and samples are available at GitHub: https://github.com/DynamicGraphSystem/StructuralStability 


\section{Funding}

This work was partially supported by FEDER Ministerio de Economía, Industria y Competitividad grants PGC2018-096540-B-I00 and PGC2018-098308-B-I00, and Proyecto I+D+i Programa Operativo FEDER Andalucia US-1254251.

\section{Acknowledgments}

Authors thank Prof. Francisco J. Esteban, at the Faculty of Biology at Jaen University (Spain) for their useful suggestions to improve a previous version of this paper. We also want to thank the Computational Center at the Computer Engineering High Technical School at Seville University and Jesús Cano for technical asistence.

\section{References}

1. Greene D, Doyle D, Cunningham P. Tracking the Evolution of Communities in Dynamic Social Networks. In: Proceedings of the 2010 International Conference on Advances in Social Networks Analysis and Mining. ASONAM '10. Washington, DC, USA: IEEE Computer Society; 2010. p. 176-183. Available from: http://dx.doi.org/10.1109/ASONAM.2010.17.

2. Markovitch O, Krasnogor N. Predicting species emergence in simulated complex pre-biotic networks. PLOS ONE. 2018;13(2):1-19. doi:10.1371/journal.pone.0192871.

3. Palla G, Barabasi AL, Vicsek T. Quantifying social group evolution. Nature. 2007;446(7136):664-667. doi:10.1038/nature05670.

4. Xie T, France-Lanord A, Wang Y, Shao-Horn Y, Grossman J. Graph dynamical networks for unsupervised learning of atomic scale dynamics in materials. Nature Communications. 2019;10:2667. doi:10.1038/s41467-019-10663-6.

5. Afraimovich V, Dmitrichev A, Shchapin D, Nekorkin V. Complexity functions for networks: Dynamical hubs and complexity clusters. Communications in Nonlinear Science and Numerical Simulation. 2018;55:166-173. doi:10.1016/j.cnsns.2017.07.005.

6. Dellnitz M, Molo MHv, Metzner P, Preis R, Schütte C. Graph Algorithms for Dynamical Systems. In: Mielke A, editor. Analysis, Modeling and Simulation of Multiscale Problems. Berlin, Heidelberg: Springer Berlin Heidelberg; 2006. p. 619-645.

7. Jost J. Dynamical Networks . In: Feng J, Jost J, Qian M, editors. Networks: From Biology to Theory. London: Springer London; 2007. p. 35-62. Available from: https://doi.org/10.1007/978-1-84628-780-0_3.

8. Letellier C, Sendiña Nadal I, Aguirre LA. Nonlinear graph-based theory for dynamical network observability. Phys Rev E. 2018;98:020303. doi:10.1103/PhysRevE.98.020303.

9. Strogatz SH. Exploring complex networks. Nature. 2001;410(3):268-276. doi:https://doi.org/10.1038/35065725.

10. Pirani M, Costa T, Sundaram S. Stability of dynamical systems on a graph. 53rd IEEE Conference on Decision and Control. 2014; p. 613-618. 
11. Deco G, Senden M, Jirsa V. How anatomy shapes dynamics: a semi-analytical study of the brain at rest by a simple spin model. Frontiers in Computational Neuroscience. 2012;6:68. doi:10.3389/fncom.2012.00068.

12. Boccaletti S, Latora V, Moreno Y, Chavez M, Hwang DU. Complex networks: Structure and dynamics. Physics Reports. 2006;424(4):175 - 308. doi:https://doi.org/10.1016/j.physrep.2005.10.009.

13. Csermely P, London A, Wu LY, Uzzi B. Structure and dynamics of core/periphery networks. Journal of Complex Networks. 2013;1(2):93-123. doi:10.1093/comnet/cnt016.

14. Danziger MM, Bonamassa I, Boccaletti S, Havlin S. Dynamic interdependence and competition in multilayer networks. Nature Physics. 2018;doi:https://doi.org/10.1038/s41567-018-0343-1.

15. Boccaletti S, Bianconi G, Criado R, del Genio CI, Gomez-Gardeñes J, Romance $\mathrm{M}$, et al. The structure and dynamics of multilayer networks. Physics Reports. 2014;544(1):1 - 122. doi:https://doi.org/10.1016/j.physrep.2014.07.001.

16. Guimarães Jr PR, Pires MM, Jordano P, Bascompte J, Thompson JN. Indirect effects drive coevolution in mutualistic networks. Nature. 2017;550:511 - 514. doi:https://doi.org/10.1038/nature24273.

17. Bascompte J, Jordano P, Olesen JM. Asymmetric Coevolutionary Networks Facilitate Biodiversity Maintenance. Science. 2006;312(5772):431-433. doi:10.1126/science.1123412.

18. Bascompte J, Jordano P. The Structure of Plant-Animal Mutualistic Networks. In: Ecological networks : linking structure to dynamics in food webs / editors, Mercedes Pascual, Jennifer A. Dunne. Santa Fe Institute studies in the sciences of complexity. Oxford, UK: Oxford University Press; 2006. p. 143-159.

19. Bascompte J, Jordano P. Plant-Animal Mutualistic Networks: The Architecture of Biodiversity. Annual Review of Ecology, Evolution, and Systematics. 2007;38(1):567-593. doi:10.1146/annurev.ecolsys.38.091206.095818.

20. Naimzada AK, Stefani S, Torriero Ae. Networks, Topology and Dynamics. Theory and Applications to Economics and Social Systems. Lecture Notes in Economics and Mathematical Systems. Springer-Verlag Berlin Heidelberg; 2009. Available from: https://www.springer.com/gp/book/9783540684077.

21. Saavedra S, Stouffer DB, Uzzi B, Bascompte J. Strong contributors to network persistence are the most vulnerable to extinction. Nature. 2014;.

22. Bastolla U, Fortuna MA, Pascual-García A, Ferrera A, Luque B, Bascompte J. The architecture of mutualistic networks minimizes competition and increases biodiversity. Nature. 2009;458:1018-1020.

23. Bullmore E, Sporns O. Complex brain networks: graph theoretical analysis of structural and functional systems. Nature Reviews Neuroscience. 2009;10. doi:https://doi.org/10.1038/nrn2575.

24. Deco G, Jirsa VK. Ongoing cortical activity at rest: criticality, multistability, and ghost attractors. J Neurosci. 2012;32:3366-3375. 
25. del Valle Rodríguez A, Cera M, Portillo JR. A network approach to analyze neuronal lineage and layer innervation in the Drosophila optic lobes. PLOS ONE. 2020;doi:10.1371/journal.pone.0227897.

26. Park HJ, Friston K. Structural and Functional Brain Networks: From Connections to Cognition. Science. 2013;342(6158). doi:10.1126/science.1238411.

27. Oizumi M, Albantakis L, Tononi G. From the Phenomenology to the Mechanisms of Consciousness: Integrated Information Theory 3.0. PLOS Computational Biology. 2014;10(5):1-25. doi:10.1371/journal.pcbi.1003588.

28. Esteban FJ, Galadí JA, Langa JA, Portillo JR, Soler-Toscano F. Informational structures: A dynamical system approach for integrated information. PLOS Computational Biology. 2018;14(9):1-33. doi:10.1371/journal.pcbi.1006154.

29. Zenil H, Soler-Toscano F, Dingle K, Louis AA. Correlation of automorphism group size and topological properties with program-size complexity evaluations of graphs and complex networks. Physica A: Statistical Mechanics and its Applications. 2014;404:341 - 358. doi:https://doi.org/10.1016/j.physa.2014.02.060.

30. Bascompte J, Jordano P, Melián CJ, Olesen JM. The nested assembly of plant-animal mutualistic networks. Proceedings of the National Academy of Sciences. 2003;100(16):9383-9387. doi:10.1073/pnas.1633576100.

31. Guerrero G, Langa JA, Suárez A. Attracting complex networks. In: Complex networks and dynamics. vol. 683 of Lecture Notes in Econom. and Math. Systems. Springer, [Cham]; 2016. p. 309-327.

32. Jalili M, Perc M. Information cascades in complex networks. Journal of Complex Networks. 2017;5(5):665-693. doi:10.1093/comnet/cnx019.

33. Muezzinoglu MK, Tristan I, Huerta R, Afraimovich VS, Rabinovich MI. Transients versus attractors in complex networks. Internat J Bifur Chaos Appl Sci Engrg. 2010;20(6):1653-1675. doi:10.1142/S0218127410026745.

34. Molkenthin N, Rehfeld K, Marwan N, Kurths J. Networks from Flows - From Dynamics to Topology. Scientific Reports. 2014;4. doi:https://doi.org/10.1038/srep04119.

35. Hale JK. Asymptotic Behavior of Dissipative Systems. No. 25 in Mathematical Surveys and Monographs. Providence: American Mathematical Society; 1988.

36. Temam R. Infinite dimensional dynamical systems in mechanics and physics. No. 68 in Applied Mathematical Sciences. Springer; 1997.

37. Babin AV, Vishik MI. Regular attractors of semigroups and evolution equations. Math Pures et Appl. 1938;62:441-491.

38. Ladyzhenskaya OA. Attractors for semigroups and evolution equations. Cambridge University Press; 1991.

39. Kalita P, Langa JA, Soler-Toscano F. Informational Structures and Informational Fields as a Prototype for the Description of Postulates of the Integrated Information Theory. Entropy. 2019;21(5). doi:10.3390/e21050493.

40. Rohr RP, Saavedra S, Bascompte J. Ecological networks. On the structural stability of mutualistic systems. Science. 2014;. 
41. Takeuchi Y. Global Dynamical Properties of Lotka-Volterra Systems. World Scientific; 1996. Available from: https://books.google.es/books?id=HMvdm08aSycC

42. Takeuchi Y, Adachi N. The existence of globally stable equilibria of ecosystems of the generalized Volterra type. Journal of Mathematical Biology. 1980;10(4):401-415. doi:10.1007/BF00276098.

43. Norton DE. The fundamental theorem of dynamical systems. Commentationes Mathematicae Universitatis Carolinae. 1995;36(3):585-597.

44. Banavar JR, Suweis S, Maritan A. Emergence of structural and dynamical properties of ecological mutualistic networks. Nature. 2013;500:449-452. doi:10.1038/nature12438.

45. Bascompte J, Ferrera A. A structural theory of mutualistic networks. In: Theoretical Ecology: concepts and applications. Oxford University Press; 2020. p. 93-115.

46. Song SCC, Saavedra S. Rethinking the importance of the structure of ecological networks under an environment?dependent framework. Ecology and Evolution. 2018;8:6852-6859. doi:https://doi.org/10.1002/ece3.4252.

47. Song C, Saavedra S. Structural stability as a consistent predictor of phenological events. Proceedings of the Royal Society B: Biological Sciences. 2018;285(1880):20180767. doi:10.1098/rspb.2018.0767.

48. Song C, Rohr RP, Vasseur D, Saavedra S. Disentangling the effects of external perturbations on coexistence and priority effects. Journal of Ecology. 2020;108(4):1677-1689. doi:10.1111/1365-2745.13349.

49. Guerrero G, Langa JA, Suárez A. Architecture of attractor determines dynamics on mutualistic complex networks. Nonlinear Anal Real World Appl. 2017;34:17-40. doi:10.1016/j.nonrwa.2016.07.009.

50. Murty KG. Linear Complementarity, Linear and Non Linear Programming. Sigma series in applied mathematics. Heldermann Verlag; 1988. Available from: https://books.google.es/books?id=ERhRAAAAMAAJ.

51. Fortuna MA, Stouffer DB, Olesen JM, Jordano P, Mouillot D, Krasnov BR, et al. Nestedness versus modularity in ecological networks: two sides of the same coin? Journal of Animal Ecology. 2010;79(4):811-817. doi:https://doi.org/10.1111/j.1365-2656.2010.01688.x.

52. Spearman C. The Proof and Measurement of Association between Two Things. The American Journal of Psychology. 1904;15(1):72-101.

53. McKay BD, Piperno A. Practical graph isomorphism, $\{\mathrm{II}\}$. Journal of Symbolic Computation. 2014;60(0):94 - 112. doi:http://dx.doi.org/10.1016/j.jsc.2013.09.003.

54. Snow BK, Snow DW. The Feeding Ecology of Tanagers and Honeycreepers in Trinidad. The Auk. 1971;88(2):291-322.

55. Kakutani T, Inoue T, Kato M, Ichihashi H. Insect-flower Relationship in the Campus of Kyoto University, Kyoto : An Overview of the Flowering Phenology and the Seasonal Pattern of Insect Visits. Contributions from the Biological Laboratory, Kyoto University. 1990;27:465-521. 
56. Jordano P. Database M_SD_20 Location: Nava Correhuelas. S. Cazorla, Andalusia, SE Spain. http://www.web-of-life.es/ (unpublished);.

57. Blüthgen N, Stork N, Fiedler K. Bottom-up control and co-occurrence in complex communities: Honeydew and nectar determine a rainforest ant mosaic. Oikos. 2004;106:344 - 358. doi:10.1111/j.0030-1299.2004.12687.x.

58. Bavelas A. A Mathematical Model for Group Structures. Human Organization. 1948;7(3):16-30. doi:10.17730/humo.7.3.f4033344851gl053.

59. Freeman LC. Centrality in social networks conceptual clarification. Social Networks. 1978;1(3):215 - 239 . doi:https://doi.org/10.1016/0378-8733(78)90021-7.

60. Sun J, Tang J. A Survey of Models and Algorithms for Social Influence Analysis. In: Aggarwal CC, editor. Social Network Data Analytics. Boston, MA: Springer US; 2011. p. 177-214. Available from: https://doi.org/10.1007/978-1-4419-8462-3_7

61. Newman MEJ. Modularity and community structure in networks. Proceedings of the National Academy of Sciences. 2006;103(23):8577-8582. doi:10.1073/pnas.0601602103.

62. Csardi G, Nepusz T. The igraph software package for complex network research. InterJournal. 2006;Complex Systems:1695.

63. Berge C. Fractional Graph Theory. ISI lecture notes. Macmillan; 1978. Available from: https://books.google.es/books?id=LD9PHAAACAAJ.

64. Scheinerman ER, Ullman DH. Fractional graph theory: a rational approach to the theory of graphs. Courier Corporation; 2011.

65. Patterson B, Atmar W. Nested subsets and the structure of insular mam-malian faunas and archipelagos. Biological Journal of the Linnean Society. 1986;28:65-82. doi:https://doi.org/10.1111/j.1095-8312.1986.tb01749.x.

66. Almeida-Neto M, Guimaraes P, Guimaraes Jr PR, Loyola RD, Ulrich W. A consistent metric for nestedness analysis in ecological systems: reconciling concept and measurement. Oikos. 2008;117(8):1227-1239.

67. Grimm A, Tessone CJ. Detecting Nestedness in Graphs. In: Cherifi H, Gaito S, Quattrociocchi W, Sala A, editors. Complex Networks \& Their Applications V Proceedings of the 5th International Workshop on Complex Networks and their Applications (COMPLEX NETWORKS 2016), Milan, Italy, November 30 December 2, 2016. vol. 693 of Studies in Computational Intelligence. Springer; 2016. p. 171-182. Available from: https://doi.org/10.1007/978-3-319-50901-3_14.

68. Jonhson S, Dominguez-García V, Muñoz MA. Factors determining nestedness in complex networks. PLoS One. 2013;8(9):e74025 1-7.

69. R Core Team. R: A Language and Environment for Statistical Computing; 2017. Available from: https://www.R-project.org/.

70. Beckett S, Boulton C, Williams H. FALCON: a software package for analysis of nestedness in bipartite networks. F1000Research. 2014;3(185). doi:10.12688/f1000research.4831.1. 
71. Galeano J, Pastor JM, Iriondo JM. Weighted-Interaction Nestedness Estimator (WINE): A new estimator to calculate over frequency matrices. Environmental Modelling \& Software. 2009;24(11):1342 - 1346. doi:https://doi.org/10.1016/j.envsoft.2009.05.014.

72. Aragão-Costa ER, Caraballo T, Carvalho AN, Langa JA. Stability of gradient semigroups under perturbations. Nonlinearity. 2011;24(7):2099.

73. Aragão-Costa ER, Caraballo T, Carvalho AN, Langa JA. Continuity of Lyapunov functions and of energy level for a generalized gradient semigroup. Topol Methods Nonlinear Anal. 2012;39(1):57-82.

74. Carvalho A, Langa JA, Robinson J. Attractors for infinite-dimensional non-autonomous dynamical systems. Applied Mathematical Sciences. Springer New York; 2012. Available from: https://link.springer.com/book/10.1007/978-1-4614-4581-4.

75. Conley C. Isolated invariant sets and the Morse index. No. 38 in CBMS Regional Conference Series in Mathematics. Providence: American Mathematical Society; 1978.

76. Stone L. The Google matrix controls the stability of structured ecological and biological networks. Nature Communications. 2016;7. doi:10.1038/ncomms12857.

77. Okuyama T, Holland JN. Network structural properties mediate the stability of mutualistic communities. Ecology letters. 2008;11(3):208-216.

78. Suweis S, Simini F, Banavar JR, Maritan A. Emergence of structural and dynamical properties of ecological mutualistic networks. Nature. 2013;500:449-452. doi:10.1038/nature12438.

79. James A PM Pitchford JW. Disentangling nestedness from models of ecological complexity. Nature. 2012;12:227-230. doi:10.1038/nature11214.

80. Pascual-García A, Bastolla U. Mutualism sup- ports biodiversity when the direct competition is weak. Nature Communications. 2017;8 (14):326.

81. Henry DB. Geometric theory of semilinear parabolic equations. Berlin: Springer-Verlag; 1981.

82. Bortolan MC, Carvalho AN, Langa JA. Attractors under autonomous and non-autonomous perturbation. vol. 246 of Mathematical Surveys and Monographs. American Mathematical Society Providence RI; 2020. Available from: https://bookstore.ams.org/surv-246/.

83. Cross GW. Three types of matrix stability. Linear Algebra and its Applications. 1978;20(3):253 - 263. doi:https://doi.org/10.1016/0024-3795(78)90021-6. 


\section{Supplementary Material}

\section{S1. Attractors as informational structures}

In [49] authors develop a full mathematical study of system (1). In particular, they show sufficient conditions for existence and uniqueness of solutions, so defining a dynamical system $\{T(t)\}_{t \geq 0}$ for (1) which possesses a global attractor $\mathcal{A}$. The phase space $X$ will be the space in which the dynamics takes place; in our case $X=\mathbb{R}^{n}$. We define a dynamical system on $X$ as a family of non-linear operators $\{S(t)\}_{t \in \mathbf{R}^{+}}$,

$$
\begin{gathered}
S(t): X \rightarrow X \\
u \in X, \quad S(t) u \in X,
\end{gathered}
$$

which describes the forwards dynamics of each $u \in X$. In our case, $S(t) u_{0}=u\left(t ; u_{0}\right)$, the solution represents the solution of (1) at time $t$ with initial condition $u(0)=u_{0}$.

The global attractor is the central concept in dynamical system theory, since it describes all the future scenarios of the associated given phenomena. It is defined as follows 35 38, 74, 81, 82: A set $\mathcal{A} \subseteq X$ is a global attractor for $\{S(t): t \geq 0\}$ if it is

(i) compact,

(ii) invariant under $\{S(t): t \geq 0\}$, i.e. $S(t) \mathcal{A}=\mathcal{A}$ for all $t \geq 0$, and

(iii) attracts bounded subsets of $X$ under $\{S(t): t \geq 0\}$; that is, for all $B \subset X$ bounded

$$
\operatorname{dist}_{H}(S(t) B, \mathcal{A}):=\sup _{b \in B} \inf _{a \in \mathcal{A}}(S(t) b, a) \stackrel{t \rightarrow \infty}{\longrightarrow} 0 .
$$

Suppose $A$ in $(3)$ belongs to class $S_{w}$ or is Lyapunov-stable (see [83]), i.e., $A \in S_{w}$, in the sense that there exists a diagonal positive matrix $W$ such that $W A+A^{T} W$ is negative definite. In this case the whole structure of the global attractor for Lotka-Volterra systems can be characterized [31,49]. Indeed, it is known that the dynamics of (3) generates an attractor, which is a structured finite set of stationary points (or equilibria) for the system, for which there exists a globally stable stationary point. The right part of Fig. 10 represents the attractor corresponding to the graph on the left with the given $\alpha_{i}$ and $\gamma_{i j}$ parameters. Due to the informational nature of a global attractor, this attractor characterization has been defined as Informational Structure in [28. The informational structure for 11) not only informs on all the stationary points of the system, but the way they are connected, showing a precise hieralchical structure by levels of information (see Fig 10) (see also 28,39). 

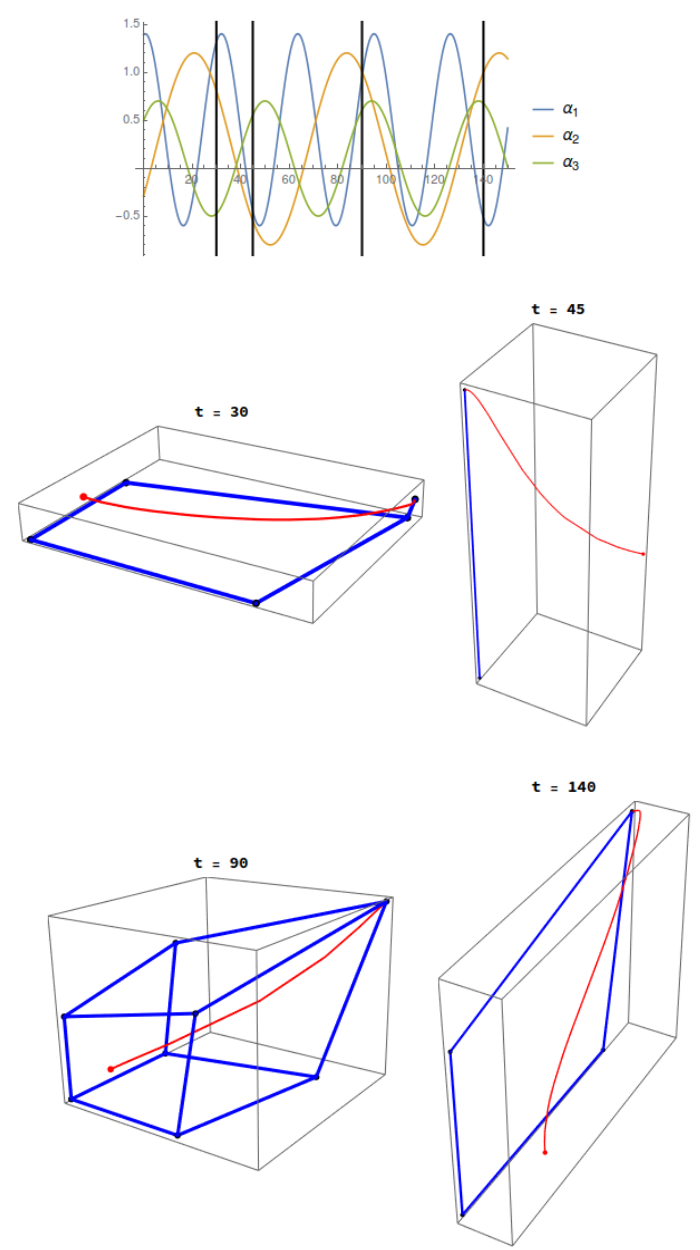

Fig 9. The evolution of four different scenarios defined over the same graph in the same state. A three-nodes graph is considered. A system of differential equations as 20 is defined for the three nodes. Here, $\gamma_{j i}=0.21$ in all cases). Below, the evolution in time of the state $(0.2,0.2,0.2)$ of the system, depending on the value of the $\alpha_{i}$ parameters which affect the behaviour of the nodes of the graph but not to its connectivity. The starting point of the (red) trajectories is always the same initial point but the trajectories are quite different. The changes in the trajectories are governed by the different Informational Structures (figures delimited by the blue lines) in each of the dynamical systems which determine the future scenarios of the system. These attractors are given by all parameters in the system. In each case, the trajectory goes to a special point which is the global stable solution in the phase space. 

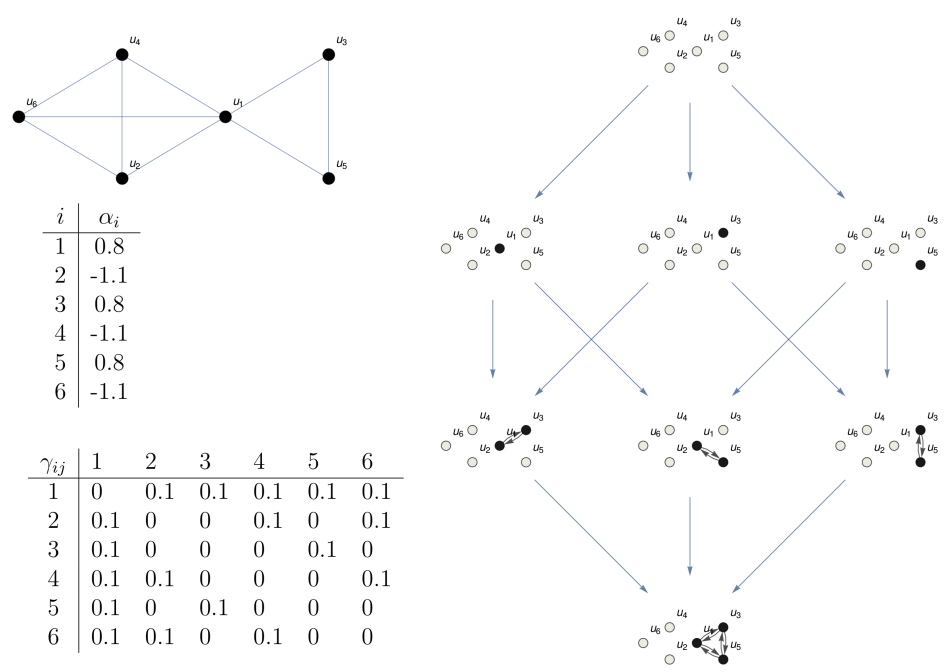

Fig 10. Informational Structure. Graph with six nodes (top left) where a dynamics is defined by means of a Lotka-Volterra cooperative system with $\alpha_{i}$ and $\gamma_{i j}$ parameters as shown in the tables. The attractor associated to the system, the Informational Structure (IS) is shown on the right. Its eight nodes correspond to non-negative stationary points in the dynamics of the system. Arrows represent solutions going to one point (limit with time approaching $-\infty$ ) to another (when time approaches $+\infty$ ). For clarity, transitive arrows are not shown. Nodes in the IS are subgraphs of the original one with vertices having a value greater than 0 in black and vertices with a value equal to 0 in grey.

\section{S2. Convex cone partition of $\mathbb{R}^{n}$.}

Let us introduce the precise definitions related to global structural stability: let $D=\{1, \ldots, n\}, I \subset D$ and $J=D \backslash I$. Let $A$ a Lypaunov-stable matrix and $B_{. j}=-A_{. j}$ for $j \in J$ and $B_{. j}=-I . j$ (negative of identity matrix) for $j \in I$, where $B_{. j}$ the $j$-column of matrix $B$. Define the convex cone as

$$
\operatorname{pos}\left(B \cdot \cdot_{1}, \ldots, B \cdot N\right)=\left\{\alpha \in \mathbb{R}^{N}: \alpha=r_{1} B \cdot 1+\ldots+r_{n} B \cdot N ; r_{i} \geq 0\right\} .
$$

Then, for any $\alpha \in \operatorname{pos}\left(B \cdot_{1}, \ldots, B \cdot N\right)$, the unique globally stable stationary solution of (3) $u_{J}^{*}=\left\{u^{*}(1), \ldots, u^{*}(n)\right\}$ satisfies (see [41)

$$
\begin{aligned}
\left\{i \in D / u^{*}(i)=0\right\} & =I, \text { and } \\
\left\{j \in D / u^{*}(j)>0\right\} & =J .
\end{aligned}
$$

This is an important result as, given any possible stationary point of the system, there exists an associated convex cone as described in $\operatorname{pos}\left(B_{\cdot_{1}}, \ldots, B \cdot_{N}\right)$ such that, when $\alpha \in \operatorname{pos}\left(B_{\cdot_{1}, \ldots, B \cdot N}\right)$, this stationary point is globally asymptotically stable ( [41]).

For instance, if we consider a 4 D Lotka-Volterra system, and $J=\{1,2\}$ (so that $I=\{3,4\}$,) the portion (i.e., the cone $C_{J}$ ) of the $\mathbb{R}^{4}$ space for parameter $\alpha$ assuring that the global asymptotic stationary point is of the form $u_{J}^{*}$ is given by

$$
C_{J}=\left\{\alpha \in \mathbb{R}^{4}: \alpha=r_{1}\left(-A_{\cdot 1}\right)+r_{2}\left(-A_{\cdot 2}\right)+r_{3}\left(-I_{\cdot 3}\right)+r_{4}\left(-I_{\cdot 4}\right), r_{i}>0\right\} .
$$

Even more interesting, the sixteen possible cones $\left(2^{4}\right) C_{J}$, for all possible $J$ - choices, form a partition of $\mathbb{R}^{4}$, i.e., the union of cones fills all the space and there is no intersection between them. 
Fig 11 shows an example of the calculation for a graph of five nodes $\left(n_{1}, n_{2}\right.$ at the left and $n_{3}, n_{4}, n_{5}$ at the right). Competition (dashed lines) is assumed between every pair of nodes on the same side of the graph and cooperation (solid lines) exists when there is a link $n_{i} \leftrightarrow n_{j}$ joining nodes of different sides. Note that when considering cooperation relationships is a bipartite graph. In general, $\beta_{i j}$ is not necessarily equal to $\beta_{j i}$ and the same for $\gamma_{i j}$ and $\gamma_{j i}$. Matrix $M$ in (6) contains all connectivity parameters. Observe that the diagonal is 1 and 0 represents no interaction between nodes. Given $M$, a certain $\bar{\alpha} \in \mathbb{R}^{N}$ is in the maximum biodiversity cone when there exist

$r_{1}, r_{2}, \cdots, r_{N} \geq 0$ verifying (7). Structural stability for $M$ is defined as the proportion of $\bar{\alpha} \in \mathbb{R}^{N}$ in the maximum biodiversity cone; i.e., the structural stability of $M$ is equal to the proportion of points of the $\mathbb{R}^{N}$ sphere of radius 1 centred at 0 in the maximum biodiversity cone.

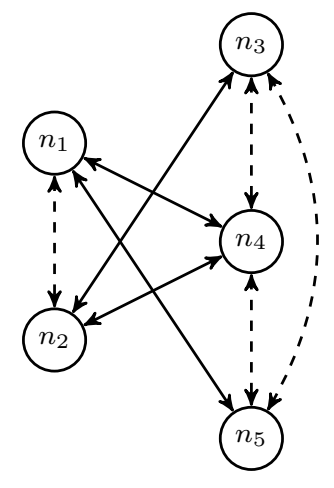

$$
\begin{aligned}
M= & \left(\begin{array}{ccccc}
1 & \beta_{21} & 0 & -\gamma_{41} & -\gamma_{51} \\
\beta_{12} & 1 & -\gamma_{32} & -\gamma_{42} & 0 \\
0 & -\gamma_{23} & 1 & \beta_{43} & \beta_{53} \\
-\gamma_{14} & -\gamma_{24} & \beta_{34} & 1 & \beta_{54} \\
-\gamma_{15} & 0 & \beta_{35} & \beta_{45} & 1
\end{array}\right) \\
& \sum_{i=1}^{5} r_{i}\left(\begin{array}{c}
m_{1 i} \\
m_{2 i} \\
\vdots \\
m_{5 i}
\end{array}\right)=\left(\begin{array}{c}
\alpha_{1} \\
\alpha_{2} \\
\alpha_{3} \\
\alpha_{4} \\
\alpha_{5}
\end{array}\right), \quad r_{i} \geq 0
\end{aligned}
$$

Fig 11. The cone of maximum biodiversity and structural stability. Left: Example $(2+3)$-bipartite graph with two nodes on the left and three nodes on the right.

Competition (dashed lines) is between all elements on the same side, while cooperation occurs between elements on different sides for which there is an edge represented by a solid line. Cooperative relationships form a bipartite graph with two groups of nodes (left and right). Top right: Connectivity matrix $M$ of the system. Competition parameters $\beta_{i j}>0$ are set for all pairs of nodes $n_{i}, n_{j}$ in the same group (both at the left or the right). Cooperation parameters $\gamma_{i j}>0$ exist for nodes $n_{i}, n_{j}$ in different groups (one in the left and the other in the right) only when there is an arrow $n_{i} \leftrightarrow n_{j}$ in the graph. In our experiments $\beta_{i j}=\beta_{j i}$ and $\gamma_{i j}=\gamma_{j i}$. Bottom right: Equation to determine if a given point $\bar{\alpha}=\left(\alpha_{1}, \cdots, \alpha_{5}\right) \in \mathbb{R}^{5}$ is in the maximum biodiversity cone. If there exist some $r_{i} \geq 0,1 \leq i \leq 5$, which verify (7), then $\bar{\alpha}$ is in the maximum biodiversity cone of $M$. To ensure the existence of a solution, the sum of the absolute values of each row or column of $M$ (including the 1 in the diagonal) must be always less than 2. This is equivalent to bound to 1 the sum of weights of all edges adjacent to each node of the graph (node degree bounded to 1). Given $M$, structural stability is defined as the proportion of points $\bar{\alpha} \in \mathbb{R}^{5}$ for which $(7)$ has a solution. Since the cones are centred at 0 , considered points can be limited to those on the surface of the $\mathbb{R}^{5}$ sphere of radius 1 centred at 0 . The estimation of the structural stability is done by the Monte Carlo method.

The global structural stability of a system allows to study bifurcation and transitions between different biodiversity scenarios. Indeed, the borders, which are now mathematically well defined, of each cone are critical zones for the sudden transition to one biodiversity scenario to a different one. Moreover, we can also observe in a global way the dependence of the cone partition on the parameters of the system (see Fig 12). 
$\mathbf{A}$

Evolution by changing $\gamma_{23}$

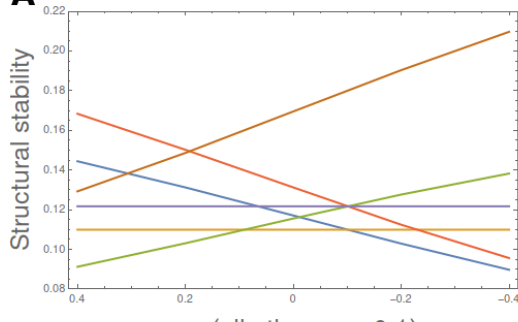

$\gamma_{23}$ (all other $\gamma_{\mathrm{ij}}=0.1$ )

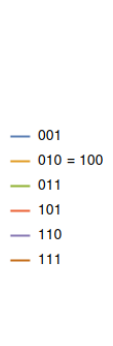

C

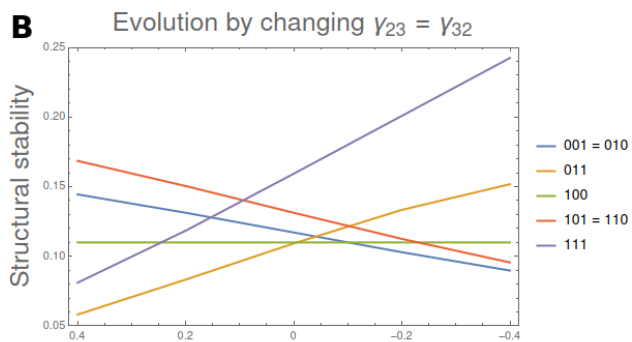

$\gamma_{23}, \gamma_{32}\left(\right.$ all other $\gamma_{i j}=0.1$ )

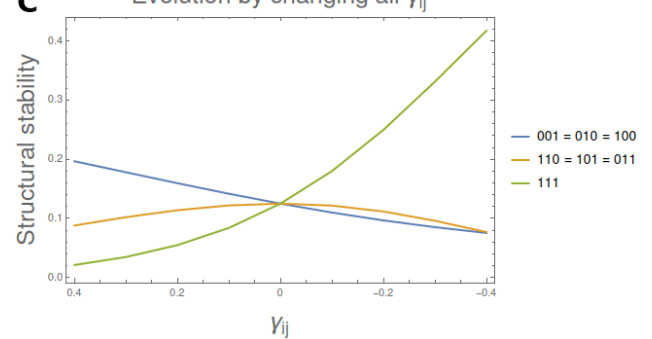

Fig 12. Evolution of Global Structural Stability on parameters on a 3D LV system. A. We observe the evolution of the size of the cones when we changing parameter $\gamma_{23}$ from competition (0.4) to cooperation values $(-0.4)$. We observe that cones of maximal biodiversity $\left(u_{111}\right)$ and the cone associated to stationary point $u_{011}$ behaves monotonically increasing with $\gamma_{23}$. B. The same result by now changing simultaneously $\gamma_{23}, \gamma_{32}$. Note that the cones for $u_{011}$ and $u_{111}$ now grow faster, while other cones with constant size now decreases (as those associated to $u_{010}$ and $u_{110}$ ). C It is shown the Global Structural Stability from a competitive system to a cooperative one, by changing all the parameters in matrix $A$. Note that, among all, it is the cone with maximal biodiversity the only one increasing with an income of cooperation in the system. 